\title{
Work in Progress: Students' Informal Reasoning when Approaching Classroom- based Scenarios Involving Diversity and Inclusion Issues
}

\section{Darby Rose Riley, Rowan University}

Darby Riley is a student of mechanical engineering at Rowan University. She has a special interest in education, as well as issues of diversity and inclusion, both of which have pushed her to seek leadership positions wherever possible. She is a founding member of Rowan University's oSTEM chapter, and working to make Rowan's campus a safe and welcoming place for all students. Darby hopes to pursue a graduate's degree in engineering education or related fields of social and psychological sciences.

\section{Joshua Bourne Reed, Rowan University}

Josh Reed is a mechanical engineering student at Rowan University. He is the student government representative for the IEEE Women in Engineering Rowan Student branch and volunteers teaching engineering at local middle schools. He also holds a leadership position in the Bantivoglio Honors Concentration in the Honors College. Josh is very passionate about education as well as the social issues in both the engineering and education systems. He hopes to further his understanding in both of these fields.

\section{Dr. Richard T. Cimino, Rowan University}

Dr. Richard T. Cimino is a Lecturer in the Experiential Engineering Education Department (ExEEd) at Rowan University. He received his Ph.D in Chemical \& Biochemical Engineering from the Rutgers University, with a focus in adsorption science and the characterization of porous materials. His research interests include engineering ethics and broadening inclusivity in engineering, especially among the LGBTQ+ community. His funded research explores the effects of implicit bias on ethical decision making in the engineering classroom. Dr. Cimino has published papers and given presentations at national and international engineering conferences. He teaches Freshman and Sophomore Engineering Clinics at Rowan University.

\section{Dr. Stephanie Farrell, Rowan University}

Dr. Stephanie Farrell is Professor and Founding Chair of Experiential Engineering Education at Rowan University (USA). Prior to 2016 she was a faculty member in Chemical Engineering at Rowan for eighteen years. Dr. Farrell has contributed to engineering education through her work in inductive pedagogy, spatial skills, and inclusion and diversity. She has been honored by the American Society of Engineering Education with several teaching awards such as the 2004 National Outstanding Teaching Medal and the 2005 Quinn Award for experiential learning, and she was 2014-15 Fulbright Scholar in Engineering Education at Dublin Institute of Technology (Ireland)tephanie Farrell is Professor and Founding Chair of Experiential Engineering Education at Rowan University (USA) and was 2014-15 Fulbright Scholar in Engineering Education at Dublin Institute of Technology (Ireland).

\section{Dr. Cheryl A. Bodnar, Rowan University}

Cheryl A. Bodnar, Ph.D., CTDP is an Assistant Professor in the Department of Experiential Engineering Education at Rowan University. Dr. Bodnar's research interests relate to the incorporation of active learning techniques in undergraduate classes as well as integration of innovation and entrepreneurship into the engineering curriculum. In particular, she is interested in the impact that these tools can have on student perception of the classroom environment, motivation and learning outcomes. She obtained her certification as a Training and Development Professional (CTDP) from the Canadian Society for Training and Development (CSTD) in 2010, providing her with a solid background in instructional design, facilitation and evaluation. She was selected to participate in the National Academy of Engineering (NAE) Frontiers of Engineering Education Symposium in 2013 and awarded the American Society for Engineering Education Educational Research Methods Faculty Apprentice Award in 2014. 


\section{Work In Progress: Students' Informal Reasoning when Approaching Classroom Based Scenarios involving Diversity \& Inclusion Issues}

\section{Introduction}

When encountering complex and ill-defined problems, the decision-making process is often almost entirely guided by informal reasoning [1]. Informal reasoning is the process used when encountering troublesome scenarios with no "true" solution, such as those found in engineering design prompts and social or ethical dilemmas. A study conducted by Sadler and Zeidler [2] identifies three specific approaches to informal reasoning, defined as emotive, rationalistic, and intuitive. Emotive reasoning applies empathy towards others and relates to the feelings of those impacted in a given scenario. A rationalistic approach takes a more logical stance that weighs the consequences of actions, and often employs arguments based in factual evidence gathered from observed details. Finally, an intuitive approach is based on the initial reaction of a person to the scenario, often referred to colloquially as a "gut reaction." Sadler and Zeidler [2] state that all three of these approaches intermingle, sometimes even being used in combination with each other, in the process of informal reasoning.

Research has shown that unethical behavior can arise when individuals do not recognize their behavior as unethical. Sezer et al. [3], explored the conditions under which "ethical blind spots" result in unintentional unethical behavior. Their work focused on three types of ethical blind spots that can cause individuals to act against their conscious ethical values: (1) implicit biases, (2) temporal distance from the ethical dilemma and (3) decision biases that result in disregarding or mis-evaluating others' ethical lapses In educational contexts, unintended biases have a significant effect on classroom dynamics, student learning, and student achievement [5].

The work presented in this paper is part of a larger study that will explore how ethical blind spots influence ethical decision making in classroom situations. As a first step, this paper focuses on understanding students' informal ethical reasoning in classroom situations involving diversity and inclusion issues by answering the following research question: What types of informal reasoning do students apply when faced with classroom based ethics scenarios related to diversity and inclusion?

\section{Methods \\ Study Design}

Eleven first-year engineering students participated in a think-aloud study where they talked through a total of six different scenarios focused on different elements of diversity and inclusion in the classroom. For the purpose of this paper, we will only provide the results from one scenario. The scenario was as follows: 
You have been informed by your Freshman Clinic Professor that a guest speaker from Gold Corporation will be invited to speak during class next week. This speaker will be providing information on how freshman engineering students can gain access to internship opportunities in their first year. However, you've been made aware that this speaker has a long history of expressing anti-LGBTQ+ views. Do you think that this speaker should be allowed to present? Yes, No, or Can't Decide.

Following each scenario, students were prompted to respond to a series of questions designed to draw out how they used each type of informal reasoning when making their decision using a protocol developed by Sadler and Zeidler [2]. A senior and junior member of the research team were present at the start of each protocol to respond to any questions or concerns the student may have after which time the senior member of the research team left the room to avoid potential impacts of a power dynamic on the data being collected. All think aloud protocols were audio recorded and then later transcribed by a third party. In addition, the students each responded to a short demographic questionnaire in which they were asked to self-identify (open-ended) their racial identity, ethnicity, gender, sexual orientation, and religious/spiritual identity. They were also asked the following two close-ended (yes/no) questions:

1. Are you working to pay for college(circle one)? If so, are you working part-time or full time?

2. Are you dealing with any mental health conditions that you feel affect your performance in school?

Each of these identity and situational questions are linked thematically to the six ethical scenarios, and will be used to study the influence of self-identity and situational factors on ethical blind spots in the students' responses. This analysis will be conducted at a later time once all of the data collected from the think aloud protocol has been analyzed to avoid any potential biases in the analysis process. Proper human subjects' approval was obtained before the conduct of this study.

\section{Data Analysis}

In analyzing transcripts, holistic coding was applied to understand the subject responses to the scenario as a whole, rather than line-by-line [6]. In this study, the "whole" refers to a subject's entire response to a single scenario. Two researchers reviewed each individual's response and summarized the emergent theme using a one-to-three word phrase that led to the creation of a code book. The transcripts were then read through in their entirety by four members of the research team and codes were applied to each subject using the prescribed code book. Provisional coding was used to analyze the strategies students applied when making their decisions using the forms of informal reasoning identified by Sadler and Zeidler [2]. Three members of the research team conducted the provisional coding of each transcript. Following the initial coding process, all five members of the research team met together to discuss any discrepancies and decide upon the final applicable codes. 


\section{Research Quality Considerations}

Researchers referenced the Quality in Qualitative Research (Q3) Framework developed by Walther, Sochacka, and Kellam [7] when preparing for collecting and analyzing their data. Examples of steps taken to ensure high quality data and analysis included the review and use of prior work in informal reasoning by Sadler and Zeidler [2] (theoretical and pragmatic validation). The researchers carefully constructed the think aloud protocol questions and developed a protocol that strived to prevent the presence of a power dynamic in the data collection process (procedural validation). First-year engineering students were included in the think aloud protocol which was designed to allow for the subjects to freely share their thoughts and ideas and provide the students with the option to change their decision at any point in the think aloud protocol (communicative and pragmatic validation). All researchers had the opportunity to discuss the coding when making decisions on discrepancies to ensure better quality of results (procedural and communicative validation). Any changes that were made to the protocol during the data collection and analysis process were kept in an audit trail (process reliability).

\section{Results and Discussion}

Analysis of the student responses yielded the following set of themes. In parentheses are the number of student responses $(\mathrm{N}=11$ total) that were coded as the corresponding theme. A. Acknowledging Complexity (1): Subjects discuss details of the situation, oftentimes using the fact that the situation is complicated to support their point. Their decision is typically that which is the least invasive (can be any of the three depending upon scenario), and use phrases such as "there's a lot to consider."

B. Broader Scope (2): Subject is focused on the diversity/inclusivity issue highlighted in the scenario. The subject applies the consequences of the scenario to a broader scope than the specific situation. They look at how situations like this affect not only the people at that specific time, but also after the fact and how it affects the community as a whole.

C. Compartmentalizing (5): Subjects agree that there is an issue related to diversity/inclusion, but it is irrelevant to the decision at hand. Often saying things like "In general, this is inappropriate. In this situation..."

E. Equivocating (1): Subject is focused on having a back-and-forth with themselves, often bouncing between two (or more) alternate perspectives. Usually in a "can't decide" scenario, but can become prevalent through the questioning process.

S. Solution-Focused (2): Students tend to craft their own "decisions" which revolve around subtleties in action and, although they may fall into "yes" or "no" broader categories, may select a course of action which is entirely outside of these options. Often uses phrases such as "what I would do is..."

Results from the coding were compared across the researchers to determine the inter-rater reliability associated with this portion of the coding process. Inter-rater reliability is used as a measure to determine the relative consistency that exists between raters when changing an 
open-ended coding process into quantitative scores as shown above [8]. An intra-class correlation coefficient using the "average measure" function in SPSS with 4 raters across 11 subjects was found to be 0.901 with a $95 \%$ confidence interval ranging from 0.855 to 0.955 which can be taken to represent good to excellent reliability [9].

The most frequently observed code was "Compartmentalizing," in which subjects would attempt to separate complex issues into smaller, more manageable pieces to isolate the situation at hand from the more difficult ethical questions that the scenario may have implied. This type of response is most clearly demonstrated by the following quote from Subject 496:

"[T]here's no solid justification knowing that his views are going to change how he presents his presentation."

All other subjects coded as "Compartmentalizing" expressed a similar sentiment; that there exists a separation from the issue of diversity/inclusivity implied and the question posed.

Responses that reflected the second most frequent codes, "Broader Scope," and "Solution-Focused" focused on the diversity/inclusivity issue implied in the scenario and either applied the proposed solution to other, similar issues (broader scope) or tried to find a compromise between the parties involved (solution focused).

Subject 719: Broader Scope "...[H] aving our school, our university associating with that person could make other people feel, think that the school associates with those views."

Subject 539: Solution-Focused "...I would offer to talk to the professor about my feelings towards the speaker coming, and then I would also offer if the speaker's not speaking for the entire class, to excuse myself, to say I'm going to the restroom, or such, during the time that the speaker is in the classroom...."

The other responses tended to fall somewhere between these three approaches, often using both the connection to/the separation from diversity and inclusivity in different measures to support their points. The "Equivocating" and "Acknowledging Complexity" codes are especially demonstrative of this reasoning strategy, and responses that were coded as "Equivocating" or "Acknowledging Complexity" also tended towards less decisive responses.

Rationalistic reasoning was dominant across all student responses (86 out of 132 total coded phrases or $65 \%$ ). This result agrees with Sadler and Zeidler, who observed that a rationalistically-dominant outcome is expected [10] for most situations. Rationalistic responses were even found to questions that were supposed to elicit emotive responses. For example, after being asked "In arriving at your decision, did you take into consideration the feelings or perspectives of anyone else? If so, how did this affect your decision making?", Subject 155 responded:

"I took into consideration the kids who I guess ... who, who are LGBTQ+ or who have people in their lives who are close to them who are...then I looked at the perspective or I guess the, the benefits and downfalls of the class as a whole..."

The emotive response was observed less often (39 out of 132 coded phrases or $30 \%$ ), usually in one of two ways; first, subjects would project their own feelings onto the scenario characters, 
usually through a personal connection of some sort. In some cases, this manifested as a general statement of similarity, such as Subject 155's expression of empathy:

"I have a lot of friends that are gay. I have a few that are bi, um, and I, I'm, I've heard them talk about some people who are very anti-LGBTQ+ and, uh, not accepting and I mean it, it hurts but a lot of the time..."

In other cases, subjects told a personal story which they felt related to the scenario. Subject 307 relayed the following story to support their point of intolerance towards hateful speech:

“...I don't, I don't like it when someone, uh, that is against any sort of community being in some sort of higher power. Um, I was in Boy Scouts for a pretty long time, and then one time, I heard my, uh, Scoutmaster say something very racist, uh, against African-Americans, and you could probably guess which word he was using. And I quit Boys Scouts that day. I did not want to be under, you know, a Scoutmaster that was believing in those kinds of views."

Lastly, the intuitive response was rarely recorded (only 7 out of 132 coded phrases or 5\%), with most intuitive responses manifested in a direct response to the prompt focused on intuitive reasoning. In the rarest case, intuitive reasoning was observed when subjects felt a connection to a certain response, and yet were unable to rationalize this response. This is best demonstrated by Subject 970's attempt to explain their own response to the scenario:

“...I don't know I feel like issues like this tend to be oversaturated in liberal decision making. Especially academic institutions. You know? Like I don't know. I don't really know how to explain this."

The relative lack of intuitive reasoning is believed to be a limitation of the questioning process, discussed further below.

\section{Limitations}

One limitation of this study was the small sample size of individuals that participated in the study. Although we attempted to recruit 15 first-year engineering students to participate, we weren't able to obtain this final number based on student interest. There were also limitations in the think aloud protocol itself that didn't seem to provide adequate means for eliciting an intuitive response despite providing an opportunity for subjects to express this form of reasoning. We also acknowledge that our study was performed only with first-year engineering students at one institution, which may limit the transferability of the results presented. We do believe that these results show some important trends, but it will be necessary for follow-up studies to be performed at other institutions to verify that the results are transferable to other engineering classroom contexts.

\section{Conclusions}

There exists a divide in the overall problem-solving approaches students undertake to engineering classroom scenarios. Some students aim to simplify the problem through compartmentalization, while others prefer to explore the greater context of the scenario. The most common approach was to compartmentalize the diversity and inclusivity aspects of the 
scenarios presented to them. This is believed to be tied to the divide between formal and informal reasoning; by compartmentalizing aspects of the given scenario, students are able to turn a complex problem requiring informal reasoning into a simpler problem which can be solved through logic-driven formal reasoning alone. The balance of informal reasoning processes within each approach remained approximately the same for all responses. Rationalistic reasoning dominated, emotive reasoning was second, and intuitive was rarely observed at all.

It is hoped that this research can be used to enhance students' awareness of their own decision-making processes, allowing for a greater insight into their own values, ethics, and morals. Future work will include analyzing the data collected on informal reasoning with the demographic questionnaire data, in the hopes of understanding how informal reasoning is affected by personal identity and situational factors. This will be immensely useful in creating a first-year engineering experience that promotes the teaching of ethics and ethical decision-making. When complete, the results from this work will be used to guide the development of ethics-based curriculum content, as well as promote classroom discussions and introspection on the part of students.

\section{References}

[1] T. Sadler, "Informal reasoning regarding socioscientific issues: A critical review of research," Journal of Research in Science Teaching, vol. 41, no. 5, pp. 513-536, 2004.

[2] T. Sadler and D. Zeidler, "Patterns of informal reasoning in the context of socioscientific decision making", Journal of Research in Science Teaching, vol. 42, no. 1, pp. 112-138, 2004.

[3] O. Sezer, F. Gino, M. H. Bazerman, "Ethical blind spots: explaining unintentional unethical behavior," Current Opinion in Psychology, vol. 6, pp. 77-81, 2015.

[4] "Understanding Implicit Bias", kirwaninstitute.osu.edu, 2015. [Online]. Available: http://kirwaninstitute.osu.edu/research/understanding-implicit-bias/. [Accessed: 15- Oct- 2018].

[5] L. van den Bergh, E. Denessen, L. Hornstra, M. Voeten and R. Holland, "The Implicit Prejudiced Attitudes of Teachers", American Educational Research Journal, vol. 47, no. 2, pp. 497-527, 2010.

[6] M. B. Miles, A. M. Huberman, and J. Saldana,Qualitative data analysis: a methods sourcebook. Sage Publications, 1994. 
[7] J. Walther, N. Sochacka and N. Kellam, "Quality in Interpretive Engineering Education Research: Reflections on an Example Study", Journal of Engineering Education, vol. 102, no. 4, pp. 626-659, 2013.

[8] P.J. Lavrakas, "Inter-rater reliability", Encyclopedia of Survey Research Methods, Thousand Oaks, CA: Sage Publications, Inc. doi: 10.4135/9781412963947, 2008, pp.360-362.

[9] J. Fleiss, The design and analysis of clinical experiments. New York: John Wiley \& Sons, 1986.

[10] T. Sadler and D. Zeidler, "The significance of content knowledge for informal reasoning regarding socioscientific issues: Applying genetics knowledge to genetic engineering issues", Science Education, vol. 89, no. 1, pp. 71-93, 2004. 